\title{
PROCEEDINGS OF THE SOCIETY OF BRITISH NEUROLOGICAL SURGEONS: 58th MEETING
}

The 58th Meeting of the Society of British Neurological Surgeons was held at the Walton Hospital, Liverpool, on December 5 and 6, 1958. The President, G. S. Clark-Maxwell (Derby), was in the Chair. Dr. J. Poppen (Boston, Mass.) attended as the special guest of the Society.

Two Unusual Cases: Multiple Meningiomata Preceded by a Spinal Neurofibroma and "Spontaneous" Atlantoaxial Dislocation

Mr. A. Sutcliffe KerR (Liverpool) reported a case of a single woman aged 50 from whom a neurofibroma had been removed from a spinal nerve root in 1937 by Sir Geoffrey Jefferson. In 1955 she had a right-sided sensory Jacksonian epileptic attack and in 1956 investigations for further similar attacks suggested a lesion alongside the falx cerebri although the ventriculogram was difficult to interpret. A left frontoparietal craniotomy revealed two small parasagittal meningiomata and two near the Sylvian point, one being $4 \mathrm{~cm}$. in diameter. These were removed but no attempt was made to reach the lesion of the falx nor to remove another tumour which could be felt in the region of the lesser wing of the sphenoid.

The epilepsy continued at three-weekly intervals although without loss of consciousness, but in 1958 she was found to have a right homonymous hemianopia, astereognosis of the right hand, a right spastic hemiparesis, and paralysis and wasting of the left side of the tongue.

$X$-ray therapy was given, a total of about $4,200 \mathrm{r}$. being spread over the whole calvarium. It caused considerable general and neurological upset but there had been steady improvement for two months. She could now walk with support although she still had minor epilepsy and an almost complete right hemiplegia with the other neurological signs unaltered. Her vestibular reactions were normal and she was not deaf. She showed no evidence of von Recklinghausen's disease nor was there any family history of that condition.

The second case was that of a boy, born in 1940, who suffered from rheumatic fever with carditis at the age of 6 . When he was 10 years old he had some unsteadiness of the legs and a dislocation of the first on the second cervical vertebra was discovered. This was said to have been reduced and a leather collar was fitted. The boy had a further attack of rheumatic fever in 1951 and further manipulations of the neck were undertaken.

In 1953 he had increasing neurological disabilities and was treated by further manipulation, head traction, and, later, a Minerva jacket. Despite this his right arm became almost completely paralysed and all the other limbs were weak and spastic. When an occipital pressure sore had healed, skull traction was applied and parallel rib grafts were inserted between the occiput and the second, third, and fourth cervical spines after removal of the whole of the posterior arch of the atlas.

Following a severe temporary deterioration of his neurological condition he made a remarkable recovery and he resumed normal schooling two years after the operation. Unfortunately he developed cardiac failure and bacterial endocarditis and died three and a half years after the operation.

\section{Aneurysms of the Middle Cerebral Artery}

Campbell Connolly (Birmingham) discussed the changes which might occur following interruption of the middle cerebral artery or its branches in the surgical treatment of an aneurysm. Other workers had shown that large communications existed between the anterior and middle cerebral arteries and that there were smaller communications between the posterior and middle cerebral arteries. Angiograms were shown demonstrating such anastomoses. Previous writers had also noted that after occlusion of the middle cerebral arteries in dogs and monkeys there was no neurological abnormality after 24 hours provided the blood pressure was kept at normal levels. When hypotension was produced by bleeding there was severe neurological disturbance (Thompson and Smith, 1951).

Seven patients were described. In one there was an aneurysm of the middle cerebral artery on the nondominant side with an intracerebral haematoma. The haematoma was evacuated and the aneurysm excised, the main stem of the middle cerebral artery being divided $3 \mathrm{~cm}$. from its origin. Three months after operation the only neurological disability was a left homonymous hemianopia.

In six other cases main branches of the middle cerebral artery were interrupted during obliteration or excision of an aneurysm. In five of these, the aneurysm was on the dominant side and four of these had intracerebral haematomas. One patient, who had an intracerebral haematoma and was operated on in extremis, died. One was dysphasic and hemiparetic after operation but improved steadily. The remaining four suffered no increase in the pre-operative disability and improved steadily.

It was considered that temporary clamping of the main stem of the middle cerebral artery proximal to an aneurysm was dangerous as, should bleeding occur, it would consist 
entirely of blood supplied from collateral arteries so that the part of the brain supplied by the middle cerebral artery distal to the clamp would be virtually exsanguinated. Severe brain damage might, therefore, be caused in a very short period of time.

It was concluded that in attacking aneurysms of the middle cerebral artery directly efforts should be made to preserve the vessel and all its branches, but, should it be necessary to sacrifice a branch, there was a good chance that no serious neurological disability would result.

\section{REFERENCE}

Thompson, R. K., and Smith, G. W. (1951). Trans. Amer. neurol. Ass., 76, 203.

\section{Intracranial Haemorrhage in Childhood}

C. B. Sedzimir (Liverpool) presented a review of intracranial haemorrhage occurring in children under 16 in a five-year period 1954-58. The material was drawn from neurosurgical, cardio-paediatric, and general paediatric services in the Liverpool area. During this period 26 children were found to have had intracranial haemorrhages. The causative diseases did not differ materially from those found in adult patients, Aneurysm was the commonest cause and was present in 11 patients, all but one over the age of 10 . The site of the aneurysm had much the same distribution as in adults. Five patients had arteriovenous anomalies, the youngest patient being under 2 years of age. In one patient an intracerebral haematoma and in five others subarachnoid haemorrhage occurred without investigation disclosing any aetiological factor. In all these patients multiple carotid and vertebral angiograms were done in an attempt to find a cause. In three patients intracranial bleeding was associated with blood dyscrasia, leukaemia in two, and purpura in one. In two patients the intracranial bleeding was associated with coarctation of the aorta; in one of them both lesions were treated surgically with good results.

\section{Brain Changes in Fatal, Ruptured, Intracranial Aneurysm}

B. E. Tombinson (Newcastle) described the brain changes in 32 fatal cases of ruptured intracranial aneurysm. At necropsy, the brain was only examined externally and the ruptured aneurysm identified where this was possible without disturbance of blood within fissures or sulci. After fixation, the vessels were dissected off and examined and large slices of brain made to study the distribution of the blood; multiple histological sections were examined.

Multiple vascular lesions were found in 15 instances (46\%); in 14 multiple aneurysms were present, and in one, an angioma in addition to an aneurysm. The site of the aneurysms was similar to those reported in other series. Two small subdural haematomas were seen.

Surface subarachnoid bleeding was sometimes scanty when large collections of blood (subarachnoid haematomata) were present between the frontal lobes, in the Sylvian fissures, or in the depths of sulci. Nine very large collections of blood, partially, or entirely confined to the subarachnoid space, were seen $(28 \%)$. Three were in the
Sylvian fissure, from middle cerebral aneurysm rupture; two had caused limited intracerebral bleeding by breaching cerebral tissue at a distance from the aneurysm, one by opening up the deep limits of a sulcus on the insular cortex. Six subarachnoid haematomata resulted from rupture of anterior cerebral or anterior communicating aneurysms. In four of these the blood lay largely between the frontal lobes; in one it lay over the infarcted corpus callosum and in one it had burrowed extensively into the olfactory sulcus. In five of these instances massive infarctions were present. Several other instances of smaller localized collections of blood were seen.

Intraventricular and intracerebral haemorrhage in quantity was seen 17 times. It was considered that most resulted from localized collections of subarachnoid blood extending into sulci, or stretching brain tissue, until they extended into the brain. In only six was the aneurysm adherent to the brain.

Ischaemic lesions were found in 25 cases $(78 \%)$ and in $13(40 \%)$ they involved the whole, or a large portion, of a major artery territory. The lesions were usually, but not always, in the distribution of the artery on which the ruptured aneurysm occurred. Four massive infarctions involved the anterior cerebral artery territory, on three occasions bilaterally. In eight cases, the infarcted area was of middle cerebral artery distribution; in two the posterior cerebral artery supply was also involved.

One instance of infarction of posterior cerebral arters distribution alone was seen.

In 10 of the 13 instances of major infarction, sub arachnoid or intracranial haematomata were present.

It was thought that localized collections of blood in sulci or fissures were of importance in several ways: by acting as space-occupying lesions; by stretching cerebrat tissues until intracerebral or intraventricular haemorrhage occurred; and probably by inducing infarction. The possible mechanisms by which localized collections might produce ischaemic lesions were stretching or kinking of vessels by the haematoma; tearing of small branches passing from major vessels to the brain; and spasm induced by stretching. Simple drainage of the bleeding site might prevent some of the fatal complications.

An additional unexplained finding was granular cell degeneration of the cerebellum in four instances. In view of the absence of clinical evidence of cerebellar dysfunction in these cases and the absence of histological reaction to the degeneration, this was considered a terminal phenomenon.

\section{Mental Abnormalities following Subdural Haematoma}

F. L. DAvies (Haywards Heath) discussed the incidence and treatment of mental disorders in patients with subdural haematoma. In a recent series of 44 consecutive head injuries admitted to a neurosurgical unit 11 had subdural haematomas. These patients were selected for admission from a much larger number of head injuries occurring in the area. Of the 11 cases, four were cured and discharged home. Four were transferred to a mental hospital with various types of mental disorder. Two of these died in the mental hospital and the other two remained in hospital a year after injury. Three patients 
died at some time after operation, thus making a total mortality of $45.4 \%$. The considerable incidence of subdural haematoma in the group was thought to be related to the high average age of a population of retired persons in coastal resorts, and in the subdural group 10 of the 11 patients were over 50 years of age. The high mortality rate was also thought to be related to high age groups in which patients were unable to combat respiratory infections. Immobility in bed, often occasioned by mental disorder, may have been a contributing factor.

Previous workers had reported a low incidence of mental disorders after head injuries. Predisposing factors considered were sex (in this series all patients with mental disorders were males), arteriosclerosis, and alcohol addiction.

In the present series confusion was present in seven cases and remained a permanent feature in four. Personality disorder was found in one case and dementia in three, one complicated by aggressive hallucinations. Korsakov psychosis was found in one case.

In all cases mental abnormalities began after a unilateral or bilateral subdural haematoma or hygroma had been treated surgically. In four patients the brain did not expand after removal of the haematoma and this feature appeared to have serious significance, as three of these died, the fourth making a good recovery. The interval between injury and operation did not appear to affect the final results; one patient with the longest time interval before operation made a satisfactory recovery and another patient operated on the day after injury died four days later. It was suggested that vascular thrombosis superimposed on arteriosclerosis was responsible for the failure of the brain to expand.

The importance of adequate surgical treatment was stressed, including attempts to expand the brain by saline injections. The importance of general care was also stressed especially control of respiratory infection and adequate dietary intake. Mental treatment consisted of psychotherapy, occupational therapy, and mental and physical rehabilitation.

\section{The Temporal Approach to Tumours of the Sella Turcica in the Presence of a Prefixed Chiasma}

JAMES L. Poppen (Boston, Mass.) discussed the temporal approach to tumours of the sella turcica in the presence of a pre-fixed chiasma. He noted that for many years tumours of the sella turcica had been attacked surgically either by the frontal or transphenoidal routes (Hirsch, 1910; Jefferson, 1940), the exception being the temporal approach used routinely by Horsley in a series of 14 patients. Jefferson (1957), in the Centenary Lecture, believed that it was natural for Horsley to use this approach since his research studies on the pituitary body had been carried out mainly in the dog and monkey. The natural and easiest route in these animals was the temporal. Neurosurgeons had approached the large extrasellar extensions under a temporal lobe as one would approach a temporal lobe tumour.

The surgical management of only the tumours that involve the pituitary body, the posterior clinoids, the upper half of the clivus, and the sphenoid sinus were discussed. These lesions were limited anteriorly by the chiasm of the optic nerve, posteriorly by the pons, and superiorly by the third ventricle.

Fortunately these tumours were relatively rare. Surgical attention had been required in only $12 \%$ of their cases of pituitary adenoma since the introduction of the rotational two million volt $x$-ray apparatus. Operation was carried out only for those adenomas that were cystic or haemorrhagic or when the vision was so precarious that immediate operation was advisable. There was also the rare individual who demanded surgery rather than the slower method of $x$-ray therapy. It was also possible that the surgeon, at times, became a bit anxious to demonstrate his prowess. This was especially true since removal of the hypophysis had been given a temporary impetus by many neurosurgeons for the treatment of cancer of the breast. That particular form of treatment would, of course, find its proper place in due time.

The tendency to enlargement of pituitary adenomas was by no means uniform. They most frequently extended through the diaphragma sellae upward if the anterior and posterior clinoids were sufficiently strong and, as the tumour enlarged, the tendency was to carry the chiasm upward, thus causing early visual changes. In other patients the posterior clinoids might be abnormally thin, allowing the adenoma to follow the line of least resistance posteriorly. In this way the tumour could become of significant size before vision was affected. Certainly in these patients the chiasm was most likely to be prefixed. In a sella turcica with a thin floor and a large sphenoid sinus the tumour extended inferiorly. This, perhaps, was the reason why visual changes developed late in the course of the disease in so many of the chromophile (acromegalic) patients, because of self-decompression of the adenoma into the large sphenoid sinus as well as the ethmoid sinus. In patients with suprasellar tumours or tumours of the upper portion of the clivus and posterior clinoids, pressure signs developed before visual changes became manifest, whereas in tumours of the tuberculum sellae visual changes always developed before increased intracranial pressure occurred.

If it had been decided that surgery was mandatory, air studies and arteriograms were of particular aid in determining the approach most likely to give the best results. If the anterior cerebral arteries were displaced posteriorly and upward with only slight opening of the carotid siphon, the anterior or frontal approach was the procedure of choice. If the posterior clinoid was eroded and the anterior clinoid reasonably normal, with little or no undercutting of the tuberculum sellae, and the angiograms showed considerable opening of the carotid siphons the temporal approach was more likely to give a better exposure of the tumour. If no obstructive signs were present, he had found that encephalograms seemed to give the more accurate and definite location of the tumour and, incidentally, the approximate location of the optic chiasm. The tumours that were favourable to this approach, therefore, included craniopharyngiomas, adenomas, chordomas, meningiomas of the upper portion of the clivus and dorsum sellae, epidermoids, and the large pituitary adenomas with a prefixed chiasm. 
Following air and angiographic studies, if the tumour was thought to be in the location described, adequate pre-operative preparation, which included steroids and intravenous urea solution, was given. The patient was placed in the sitting position (tic position) and the incision outlined. The anterior limb of the incision only was opened initially. An opening was made in the inferior portion of the squamous part of the temporal bone. The dura was opened with the loose flap turned down, then sewn to the soft tissues inferiorly. A ventricular puncture of the temporal horn was made if the brain was tense. Usually the intravenous injection of urea caused the brain to shed much of its fluid content, allowing the temporal lobe to be readily elevated for sufficient exposure of the tumour. If not, the anteriorinferior portion of the temporal lobe might be sacrificed. It had been of considerable surprise to him to find that tumours which had eroded the sella turcica and destroyed the posterior clinoid were much more superficial than would be supposed, contrary to the impression received when an attempt was made to clip a vestigial aneurysm of the internal carotid artery that extended posteriorly along the course of the posterior communicating and anterior choroidal arteries. The distance from the temporal bone seemed to be much greater. This was due, no doubt, to the contour of the temporal fossa and depended on whether it was shallow or deep.

Since the width of the temporal fossa from the inferior edge of the squamous temporal bone was only 5 to $6 \mathrm{~cm}$., it could be readily seen that if the sella was ballooned out and the posterior clinoids eroded, tumours, even though extending only a slight distance laterally, made the approach relatively shallow and simple. The more inferiorly the squamous portion of the temporal bone was removed, the easier the approach. Rarely was it necessary to extend the incision that had been outlined and the bone flap turned down. If perchance the frontal approach was used initially with the impression that the chiasm was not prefixed, but on direct examination it was found to be prefixed, the inferior limb of the frontal incision might be lengthened to the upper border of the zygomatic bone and the temporal approach used. This avoided subjecting patients to an added procedure at a later date. It was also of help if bleeding that could not be properly controlled had been encountered in the tumour during the anterior approach. This was particularly true of the vessels that might be separated from the circle of Willis in a blind attempt to remove the capsule of the tumour when the anterior approach was used.

Four craniopharyngiomas had been attacked in this manner. One patient died of post-operative thrombosis of the internal carotid artery which was markedly arteriosclerotic and had a saccular aneurysm attached. No necropsy was allowed. Four adenomas were managed in this fashion; two of the patients died. Five chordomas were extensively removed with no operative deaths; and one meningioma of the clivus was also removed.

In the use of this approach it should always be remembered that since the tumour could be visualized so readily, one was inclined to excise more radically than was necessary, as was the case in the two pituitary adenomas. All of the capsule that was not adherent to $Z$ major vessels could be readily removed. The capsule that was firmly adherent to the artery should be allowed to remain intact, however, and $x$-ray treatment given subsequently. The cystic tumours presented no particular problem since the capsule peeled away readily. The third $T$ nerve must be protected, especially during coagulation of any bleeding points or during manipulation of the tumour.

It was suggested that the temporal approach be used in patients in whom the tumour extended into the third ventricle or compressed the anterior-superior portion of $\bar{G}$ the pons and in pituitary tumours with a prefixed chiasm.

\section{REFERENCES}

Hirsch, O. (1910). J. Amer. med. Ass., 55, 772. Jefferson, G. (1940). Proc. roy. Soc. Med., 33, 433. - (1957). Brit. med. J., 1, 903 .

\section{On Certain Acute Head Injuries}

DOREEN JACOBS (Liverpool) described five cases of $\overrightarrow{\vec{c}}$ acute head injury all posing diagnostic problems with $\omega$ regard to either the anatomy or pathology of the condition.

Case 1.-A man, aged 54, with a known right-sided i injury, developed rapidly a left hemiplegia associated ? with a dilating pupil on the left. An acute subdura haematoma was found in the left temporal region. He had thus sustained a contre-coup haematoma associated wi an ipsilateral hemiparesis. It was concluded that irrespective of conflicting physical signs, the side of $Q$ dilating pupil indicated the site of pathology.

Case 2. - A man, aged 20, during a five-hour period of observation after an accident, developed a dilated lete pupil. Carotid angiography disclosed a right tempora haematoma. The dilated pupil proved, later, to be pag $\vec{\theta}$ of an ophthalmoplegia presumed to be due to direet of trauma to the nerves despite its delayed onset. This case appeared to invalidate the conclusion drawn from Case 1.

Case 3.-A man sustained a head injury and was found to have unequal pupils. Examination disclosed that he had one glass eye.

Case 4.-A child, aged 5 years, sustained a relatively minor head injury and over a period of two weeks developed papilloedema and a sixth nerve paresis. She proved to have a small depressed fracture obstructing the posterior end of the sagittal sinus which had not been radiologically obvious. Angiography suggested a sagittal $\overline{0}$ sinus block. Exploration confirmed the diagnosis and relieved the condition.

Case 5. - A child, aged 9 years, presented with a calcified extradural haematoma three months after a head injury. The only physical manifestation of intracranial pathology was the development of epilepsia 8 partialis continua seven weeks after the accident. This was controlled by medication and did not recur. The diagnosis of a space-occupying lesion was made by angiography and the exact pathology found and relieved by exploratory craniotomy.

It was concluded that these cases served to emphasize $\mathrm{N}$ that patients were individuals, with individual good or $\sigma$ bad fortune, individual physical signs, and a bias against $N$ any dogmatic classification. 


\section{A Review of 700 Cases of Epilepsy}

R. H. Hannah (Liverpool) reviewed 700 cases of epilepsy. These cases had presented themselves for investigation at a neurosurgical unit over a period of 11 years. Four hundred and fifty-eight of the cases were male and 241 female. Six hundred and twenty-nine were alive, 57 dead, and 14 presumed dead. An up-to-date follow-up was available in 435 cases. The series showed a reasonable representation of all age groups. Pneumoencephalographic studies were available in 616 cases and E.E.G. reports in 463 . A family history of epilepsy was obtained in $13.1 \%$ of a sample of 595 cases.

The cases were classified on an aetiological basis and on clinical findings. On the former basis the series was broadly divided into cases showing structural changes in the brain, as shown by air studies, and those with normal pneumo-encephalograms. Those showing organic changes were subdivided into (1) those associated with spaceoccupying lesions $(68)$; (2) those having birth injuries or head injuries (122); (3) those with a focal cicatrix in the brain $(8)$; (4) those showing diffuse organic changes (153), 84 of these attributable to a variety of infections or degenerative conditions and 69 were of uncertain aetiology; (5) those associated with cerebrovascular disease (49); (6) those with congenital anomalies (20).

Those cases with no demonstrable organic change were subdivided into (1) those with generalized attacks or petit mal (174), (2) those with focal attacks (102); (3) those associated with generalized metabolic changes (4).

On clinical grounds the cases were divided into generalized, petit mal, focal motor, focal sensory, and focal psychical. There were 373 cases with generalized and 327 attacks with focal epilepsy.

The ages of onset of the seizures were considered. The highest incidence of epilepsy due to birth injury was in the first decade; due to supposed centrencephalic causes in the second decade; due to head injuries in the third decade; due to space-occupying lesions in the fifth decade; and due to cerebrovascular disease in the sixth decade.

By comparing the duration of the epilepsy in cases with and without demonstrable organic brain changes it was shown that the duration had little relationship to the degree of brain atrophy. It was suggested that the brain changes might be the cause of the epilepsy rather than the result of it.

Of the 463 cases with E.E.G. reports available, 367 were abnormal. The generalized electrical activity corresponded to generalized seizures in $81.2 \%$ of cases and focal activity to clinically focal attacks in $70.3 \%$.

In 304 cases information regarding birth was available. Of this sample, $30 \cdot 2 \%$ showed evidence of abnormality of birth. In 402 cases information was available regarding birth injuries. Fifty-three cases had a history of injury at birth but only 31 showed abnormal air studies. Seventyseven per cent, or 449 cases, had a history of infantile convulsions.

The association of epilepsy with menstruation was investigated. Ninety-three women supplied information and there was an association in 38 cases. The majority of these fell into the groups of attacks of supposed centrencephalic origin.

\section{Some Unusual Cases of Neurosyphilis}

ROBERT Hughes (Liverpool) described a series of unusual cases of neurosyphilis seen in Liverpool during the past few years.

Case 1.-A woman, 53 years, had a one-year history indicating a dementia paralytica. When first seen the pupils were normal; a fortnight after admission to hospital she developed typical Argyle-Robertson pupils. A month after chemotherapy had been begun the pupils became almost normal although the left was slightly sluggish in reaction to light and accommodation. Her mental symptoms disappeared but at a later date she had some epileptic attacks.

Case 2.-A woman, 61 years, was diagnosed as having tabes dorsalis in 1941 and had a course of 12 injections. For a period of 15 years her neurological state deteriorated, until when seen in 1946 she was unable to stand without assistance. She had gastric crises and loss of sensation in the legs below the knees. She had been seen at various hospitals throughout this period and her serological reactions were completely negative. Unfortunately no treatment had been given and when at last effective chemotherapy was started in 1956 there was no improvement.

Case 3. - A man, 29 years, had a history of one attack of unconsciousness two years before, and was seen at an out-patient clinic with early papilloedema, and a brain tumour was suspected. The eye trouble disappeared completely on anti-syphilitic treatment.

Case 4.-A man, 49 years, had shown marked mental deterioration for the past year, some evidence of a right frontal lobe lesion clinically, and an E.E.G. showed a delta focus in this area. A brain tumour was suspected but on serological reactions this was thought to be a case of dementia paralytica.

Case 5. - A woman, 21 years, described attacks of blurring of vision of the left eye and double vision during the past year, and more recently weakness of the right side of the body. Disseminated sclerosis was suspected but the condition proved to be syphilitic.

Case 6. - A man, 40 years, was admitted as an emergency in June, 1958, with an acute lesion in the right cerebral hemisphere, neck rigidity, temperature $102^{\circ}$, and large numbers of polymorphs in the cerebrospinal fluid. The E.E.G. showed a delta focus in the right hemisphere. A brain abscess was suspected but the condition proved syphilitic and responded rapidly to chemotherapy.

Case 7.-A woman, 64 years, had had cerebellar ataxia of one year's duration. At first this was thought to be a carcinomatous neuropathy as she had had an adenocarcinoma of the colon removed five years previously. The condition proved syphilitic and during the following two years there was no evidence of a recurrence of the carcinoma.

Cases 8 to 10 were instances of neurosyphilis precipitated by head injury.

He stressed that neurosyphilis was still frequently seen in his clinic and should be considered in all neurological cases.

\section{The Anatomy of Thalamic Pain}

D. BowsHER (Liverpool) presented the results of some investigations on the brain-stems of patients dying at suitable intervals after antero-lateral cordotomy, using the Nauta silver technique. These results had confirmed 
and extended the observations of previous authors on the widespread connexions of fibres from Gower's tract. Although a few fibres passed directly to the thalamus, there was a very large spino-reticular contingent ending chiefly in four zones of the ventro-medial reticular formation. Other fibres passed to the corpora quadrigemina, the inferior olive and lateral reticular nucleus, and to the sensory cranial nerve nuclei.

In one case dying 32 days after operation, preliminary and only very approximate fibre counts had been made in transverse sections stained by the method of Häggqvist. These counts suggested that, of the fibres present in Gower's tract at C.1 level of the cord, only $45 \%$ of small fibres $(2-4 \mu)$ and $70 \%$ of large $(4-7 \mu)$ reached the rostral medulla. At mesencephalic level only $12 \%$ of small and $36 \%$ of large fibres remained. These figures served to emphasize the quantitative importance of the spinoreticular element in Gower's tract.

It had already been suggested (Bowsher, 1957) that while direct spino-thalamic fibres were responsible for acute localized pain, the spino-reticular fibres were a link in a neuron chain by which diffuse, poorly localized pain or discomfort was eventually brought into consciousness. This second pain pathway bore a remarkable resemblance to the "reticular activating system", and this parallel was investigated electroencephalographically in patients with spontaneous "thalamic" pain accompanied by anaesthesia to acute pricking pain. It was found that the alpha rhythm was only desynchronized in those cases in which it was possible to evoke the diffuse pain. It was suggested that massive cortical "activation" by non-specific impulses travelling up diffuse reticulothalamo-cortical pathways was responsible for the "thalamic" type of pain.

REFERENCE
Bowsher, D. (1957). $\quad$ Brain, 80, 606.

Observations on the Injection of Xylocaine into the Frontal Lobes

P. M. O'Flanagan (Liverpool) described the results of injecting "xylocaine" into the frontal lobes as a test for determining the probable results of a leucotomy. He noted that Fulton (1951) had suggested that where the therapeutic effect of leucotomy was in doubt the area in question could be injected with local anaesthetic as a trial. In order to evaluate this procedure a pilot study had been undertaken over the past two years.

One per cent. plain "xylocaine" had been injected bilaterally into the lower medial frontal quadrant approximately $1 \mathrm{~cm}$. superior to the orbital plate and just anterior to the frontal horn of the lateral ventricle. The amounts injected varied from 2 to $9 \mathrm{ml}$. The subjects consisted of 18 psychiatric patients, including psychotics, psychopaths, and neurotics.

Complex autonomic changes occurred in $84 \%$ of the cases immediately after the second injection. No lateralization was shown. These changes included alteration in blood pressure levels, often sudden and marked, also apnoea up to 15 minutes, and unilateral or bilateral mydriasis.
Recovery of consciousness was sometimes delayed up to four hours whilst drifts in blood pressure, pulse rate, and body temperature, changes in skin coloration, and coarse muscle fasciculations were delayed responses. Independence was shown in the immediate responses to each other.

One male patient of 62 died of a cerebellar haemorrhage (5\% mortality). One patient developed a thrombosis at the head of the right caudate nucleus and another a transient coma with hemiplegia and hyperthermia four days after injection. Both patients recovered fully.

E.E.G. investigations showed the following changes. In those patients with a profound fall in blood pressure there were hypoxic rhythms. Runs of $6 \mathrm{c}$./sec. activity were seen during the recovery phase after the fall in blood pressure. Desynchronization and decrease in amplitude of the orbital rhythms were also seen.

Statistical examination disclosed that apnoea was an unfavourable factor $\left(\chi^{2}=0 \cdot 1\right.$ level of significance). The other autonomic responses showed no statistical relationship to prognosis.

Forty-seven per cent. showed no psychiatric improvement. Seventeen per cent. manifested short and equivocal improvement. Forty-five per cent. showed a loss of physical and mental symptoms lasting seven to 84 days. Of the latter group all but one were subjected to leucotomy, and $84 \%$ showed marked improvement ot recovery.

It was concluded that a definite and maintained los of symptoms with no excessive increase in extraversiof after local injection appeared to justify a leucotomy.

\section{REFERENCE}

Fulton. J. F. (1951). The Frontal Lobes and Human Behavion [Sherrington Lecture, 1951]. University of Liverpool.

\section{The Spitz-Holter Valve}

G. H. McNaB (London) described his experiences with the Spitz-Holter valve in the treatment of hydrocephalus and showed a film of the operative procedure. He noted that the valve was designed to provide a unidirectional flow of cerebrospinal fluid from the lateral ventricle to the superior vena cava at a set pressure of $50 \mathrm{~mm}$. water. The success of the new valve was due to the use of specially purified silicone rubber. This material was non-wettable and adherent to itself so that the valves could open and shut at the set pressure without loss of resilience. The slit valve caps never came in contact with blood so that the cerebrospinal fluid was retained within its own boundaries. He reported that Lawrence, working at The Hospital for Sick Children, Great Ormond Street, had investigated a series of 182 cases of hydrocephalus not subjected to any form of operation. Of these 89 had died, nine remained progressive, three were untraced, but most importantly 81 appeared to have undergone spontaneous arrest. Of these arrested cases, $75 \%$ were educable with an I.Q. of over 50 and $57 \%$ had a normal I.Q. Many suffered additional physical handicaps but $33 \%$ of the group were free from physical disability. It followed, therefore, that operative methods of treatment must offer $N$ at least $46 \%$ arrest of hydrocephalus and more than $57 \%$ 
must be able to attend a school for normal children provided their physical handicap would permit this.

He had inserted 60 of these valves over a nine-month period, $49(81 \%)$ were still working. The cause of block was aqueduct stenosis in $16 \%$, basal cistern block in $53 \%$, and Arnold-Chiari deformity in $31 \%$. In 19 cases there was an associated myelomeningocele and consequent risk of infection. Hydrocephalus was well advanced when treatment was started and the average depth of cortex was $1.7 \mathrm{~cm}$. It was noted that whilst $2 \mathrm{~cm}$. had been suggested as the minimum depth of cortex associated with normal intelligence, he had had several cases with cortex of only $0.5 \mathrm{~cm}$. and I.Q. of 85 to 100 . The average head circumference was $19 \frac{1}{2}$ in. and it was noted that with circumferences above 22 in. the cortical depth was always below $2 \mathrm{~cm}$.

Eleven deaths had occurred in the series. Five cases of Arnold-Chiari deformity died with infected clot formation in the superior vena cava and heart. Five cases of basal cistern block died, three due to septic clot formation and two from excessive loss of cerebrospinal fluid following removal of a polythene tube from spino-ureteric drainage and removal of a valve from one side to the other. One case of aqueduct stenosis died from haemorrhage and infection following ventricular taps in another hospital. In all these cases the valve was found to be in working order at necropsy and the jugular catheter free of clot.

A full description of the valve was given and illustrated with the film. It was stressed that failure was due to clot forming on the wall of the vein at the end of the jugular catheter; minor trauma to the wall and excessive mobility of the tube might be causative factors. To prevent this, the tube was now secured at its entry to the internal jugular vein by the use of a nylon junction tube and the lumen of its distal portion had been reduced.

\section{The Management of Malignant Cerebral Astrocytoma}

Phillip HARRIS (Edinburgh) gave a preliminary report on the management of 62 patients with malignant cerebral astrocytoma seen during the period November, 1955, to November, 1957, when linear accelerator (4 MeV) radiotherapy had become available. The main advantages of supervoltage radiotherapy were less skin dose, no permanent epilation, a greater dose at depth and reduced bone absorption and therefore a higher tumour dose. It was found that whole head treatment was necessary to ensure that the whole of the tumour was being irradiated.

A decision regarding investigation and treatment was made partly on the clinical course; thus the prognosis was very poor (survival under three months) if there were severe neurological deficits and no treatment was attempted. The prognosis was better if raised intracranial pressure was the presenting symptom, and better still if the latter was epilepsy. By the age of 40 in men, and 50 in women, the prognosis was poor, over the age of 60 in either sex it was usually hopeless, below 35 years it was quite good.

Cerebral angiography was helpful in diagnosis, and also in prognosis. Thus 56 patients had this test. In 25 the typical tumour circulation of a malignant astrocytoma was seen, only two of these patients survived one year; of 7 the 31 patients without a tumour circulation on angiography, nine survived one year and six others were well after a period of two years. A further three patients were well after one year and two after two years.

Kernohan's classification of astrocytomas was used (Svien, Mabon, Kernohan, and Adson, 1949). The majority of the tumours were in Grade 4. Nine without tumour circulation on angiography were in Grade 2; only one with circulation was in this grade.

Using the above criteria, 43 patients had surgical decompression (partial excision of tumour-usually by lobectomy, in 16); and 32 had a full course $(4,500 \mathrm{r}$ ) of radiotherapy, with $50 \%$ survival of six months, and $30 \%$ of one year. This compared with $18 \%$ and $10 \%$ respectively in the 263 patients with a similar type of neoplasm seen in the previous five years.

Assessment of the results of treatment was based on the clinical state, and if available, angiographic and pathological changes. In three patients, a return of raised intracranial pressure one year after decompression and radiotherapy was due to cyst formation in the tumour site; there was a good response to further surgical treatment.

The present management of these most difficult and quite common neoplasms appeared to give improved results regarding the period of useful survival.

\section{REFERENCE}

Svien, H. J., Mabon, R. F., Kernohan, J. W., and Adson, A. W. (1949). Proc. Mayo Clin., 24, 54.

\section{Some Experiences with Echo-Encephalography}

ANTHONY JEFFERSON (Sheffield) presented his experiences with the Kelvin and Hughes Mark V flaw detector specially modified by the makers in collaboration with Dr. Douglas Gordon. Initially the frequency of the emitted ultrasound was 2.5 megacycles per second. More recently better results had been obtained with a frequency of 1.5 megacycles per second.

This apparatus might be of help in detecting the presence of, or in confirming the absence of, a supratentorial mass. He had not been able to demonstrate that it had any other diagnostic potentialities.

Fifty cases had been tested to date. In all of them there had been radiological or operative verification of the findings. In 31 cases with no midline displacement a central echo was satisfactorily obtained in 25 and with difficulty in three cases. In three cases the presence of displacement was falsely indicated. In 19 cases with midline displacement this was demonstrated satisfactorily in 13 and with difficulty in one. A central echo was falsely obtained in five cases. Some examples of the results were shown.

It was concluded that the flaw detector might give helpful information but it might mislead. It was easy to learn to use but sometimes its use was a little laborious. However, he agreed with Leksell $(1956,1958)$ that it provided a potentially valuable tool-possibly even more valuable after further slight modification. Already there had been occasions when the decision to transfer a patient to the neurosurgical department had been taken at the bedside from information derived from this device. $\mathrm{He}$ 
noted that it would take time to establish the flaw detector in the diagnostic hierarchy and that the present report was in the nature of a preliminary communication designed to draw attention to the possibilities of the method. (He expressed his gratitude to Messrs. Kelvin and Hughes Ltd. who had generously loaned the apparatus.)

\section{REFERENCE}

Leksell, L. (1956). Acta chir. scand., 110, 301 - (1958;. Ibid.. 115, 255.

The Use of Paraspinal Acrylic Inlays in the Treatment of Cervical and Lumbar Spondylosis and Other Conditions

Geoffrey KNIGHT (London) discussed the use of acrylic inlays in certain spinal conditions. He suggested that in degenerative and traumatic lesions of the spine referred pain from deep ligaments affected by altered mechanical stress was often more important than pain from root compression. Acutely painful or unstable joints could be supported and overstretching of ligaments prevented by placing an inlay under the paravertebral muscles on the posterior aspects of the laminae.

A sheet of acrylic plastic ("kallodent" preparation 222 range) was used for this purpose. If this was inserted in a soft, doughy consistency it would adapt to local curvatures and harden in situ within 24 hours. This slow setting was advantageous as the spine could be supported in the optimum position for 24 hours during which time the inlay adapted itself to the required position and formed a well-fitting and firm support. Correct consistency before insertion was important and this was usually obtained 20 minutes after the mixture of $3 \frac{1}{2}$ parts of acrylic polymer with 1 part of acrylic monomer. If the plastic was inserted while still tacky or moist an excess of serous fluid might form around the inlay. This reaction had occurred in certain cases in the lumbar region but never in the cervical. This might be due to the greater amount of material inserted but the risk of reaction was minimized by using a firm, dry inlay in a completely dry bed in which absolute haemostasis had been secured.

In the cervical region inlays had successfully relieved severe referred pain from areas of spondylosis and old, painful cases of fracture dislocation, thus enabling patients to discard collars which they had had to wear continuously. The operation was conducted in the sitting position, the inlay being inserted usually over the back of the fifth, sixth, and seventh laminae on one side only, after which the head was held in neutral position for 24 hours by a plaster back-slab.

A large inlay from the level of the occipital plate to the third cervical lamina had produced a satisfactory stabilization in a case of atlanto-occipital subluxation produced by rheumatoid arthritis.

In the lumbar region inlays had been employed in patients suffering from syndromes of low back pain with referred pain in the legs produced by overstretching of ligaments resulting from abnormal mobility of a lumbar vertebra. In these patients the spine was painless only in one balanced position. Pain was provoked by movement, by jarring of the spine while travelling, by walking, and by rotation of the spine when rolling over in bed. In the lumbar region, after the insertion of an inlay into a completely dry bed, the patient was nursed flat on the back with the spine slightly extended over a pillow for the first 12 hours and was allowed up at the end of three days.

Late Results in Five Cases of Communicating Hydrocephalus Treated by Theco-peritoneal Anastomosis

A. R. TAYLOR (Belfast) reported the results in five cases of children with communicating hydrocephalus treated by theco-peritoneal anastomosis, the survivors of eight so treated in 1952 . These cases were studied by a psychologist, a psychiatrist, and a neurological surgeon. The parents had also been interviewed. They all fell into the I.Q. groups 100-110. None had gross personality disorders and all were progressing normally at school. The results had no relationship to the thickness of the surviving pallium before operation, which in one case was $0.5 \mathrm{~cm}$. One child required a second operation in his third year; the others had presumably by then balanced their cerebrospinal fluid production-absorption mechanism as they had outgrown their tubes.

Ventriculo-Peritoneal Anastomosis in the Treatment of Hydrocephalus

J. M. ROBERTSON (Glasgow) presented his experiences in the treatment of infantile hydrocephalus by ventriculoperitoneal anastomosis and discussed the value of this procedure in other neurosurgical conditions. This type of shunt was particularly useful in that it was applicable to both obstructive and communicating hydrocephalus. Much of the success of this operation was attributed to the silicone tubing used; this was elastic and pliable, did not kink or undergo any alteration in the tissues, and did not cause any tissue reaction.

The tubing, $1.5 \mathrm{~mm}$. O.D., $0.5 \mathrm{~mm}$. bore, was inserted into a lateral ventricle, usually the right, through a posterior parietal burr hole and fixed by a flanged silicone tube threaded over it and secured to the pericranium. Most obstructions in the tube were found at the distal end and all occurred within the first two months. Usually some debris was found lodged in the tube but in others no obstruction was found and usually re-positioning of the distal end of the tube in the peritoneum produced a satisfactory result. In a few cases washing through the tube with Fisher's solution by needling the tube through the skin had cleared the obstruction; in others exposure of the abdominal end of the tube had been necessary. Sepsis had been the sole cause of failure to establish drainage in the unsuccessful cases. In three of such cases the hydrocephalus was associated with a meningocele. The shunt was established first to drain the hydrocephalus and relieve tension in the meningocele. In each case infection supervened, leading to tube obstruction and finally death in the acute stage of infection. A fourth similar patient died at operation later for closure of the meningocele. The other unsuccessful case was of a child with an aqueduct obstruction still alive two and a half years after attempts at drainage were abandoned. It was concluded that it was desirable to carry out repair 
of a meningocele before drainage was attempted. The use of cortisone to prevent abdominal reactions appeared to have some value and in the past 18 months a four-week course of cortisone had been given post-operatively in all cases. In all 33 cases had bee: treated with $85 \%$ successful results.

The outstanding problem of the operation was how to allow for the child's growth. Leaving spare coils of tube in the lateral ventricle or abdomen was unsatisfactory and the use of telescoping drains had been unsuccessful. The natural stretch of the silicone tube might compensate for some degree of growth but it was hoped that many of the cases might stabilize after a period of drainage. If necessary there was no reason why the procedure should not be repeated when the child outgrew the tube.

The operation probably had some value in posti.nflammatory cases where a spinal block or raised intracranial pressure had developed. It was even possible in the active stages of tuberculous meningitis and no evidence of peritoneal involvement had followed such drainage. Eight such patients had been treated, seven of whom were still alive, and three further patients in an inactive phase had been treated, of whom two were still alive. Nine cases of pyogenic meningitis had been treated of which seven were still alive. Further conditions considered for this operation were subdural haematomata, pseudo-mening ocele, pseudo-tumour cerebri, brain-stem tumours, and those cases where ventriculo-cisternostomy had failed.

\section{BOOK REVIEWS}

Handbuch der speziellen pathologischen Anatomie und Histologie. Edited by F. Henke, O. Lubarsch, and A. Rössle. Vol. XIII : Nervous System, edited by W. Scholz. (Section 2, parts A and B. pp. 2902; 1199 figures. DM. 859.50.) Berlin: Springer-Verlag. 1958.

The appearance of section two completes the 13th volume of the Henke-Lubarsch Handbook. Part A (pp. 1,325) deals solely with inflammatory disease of the central nervous system. The principal contributions are from Ludo van Bogaert, of Antwerp, who writes on the encephalitides and poliomyelitis; Hans Schleussing, of Munich, on meningitis; Gerd Peters, of Bonn, on multiple sclerosis and other demyelinating diseases; Ernst Sträussler, of Vienna, and O. Gagel, of Nuremburg, on neurosyphilis; and S. Scheidegger, of Basle, on tuberculosis, malaria, mycotic infections, and leprosy.

Part B (pp. 1,576) contains massive contributions from three authors: F. Erbslöh, of Munich, who writes on the neuropathological manifestations of various systemic diseases, including endocrine and blood disorders; Angel Pentschew, of Washington, on the intoxications and deficiency disease; and Hans-Joachim Colmant, of Bonn, on the various forms of motor neurone disease.

The Henke-Lubarsch Handbook is uniquely huge. Volume 13, which covers the pathology of the nervous system, consists of seven large books (of which the two parts under review are the largest), occupies $14 \frac{1}{2}$ inches of shelf space, weighs $35 \mathrm{lb}$. and costs about $£ 20610$ s. It is, and is likely to remain for several years, the major work of reference for neuropathologists all over the world-if they are fortunate enough to have access to it.

Pharmakopsychologie und Psychopathologie. By Wolfgang de Boor. (Pp. xi + 291. DM. 39.60.) Berlin: Springer-Verlag. 1956.

Much gratitude is due to anyone who undertakes the tedious labour of compiling an annotated bibliography of a rapidly expanding scientific field. Dr. de Boor has carried out this arduous task, as part of his effort to produce a compendium of "pharmaco-psychology". In order to keep his text within manageable limits, he has had to select from the vast literature; it is sometimes possible to disagree with his choice, but on the whole a balanced and informative picture is offered of the state of knowledge up to 1954. The chief defects are the relatively inadequate coverage of publications in English, and the lack of critical evaluation in the accounts of what individual workers have reported: these defects are particularly evident in the section on alcohol. Dr. de Boor hopes that this edition of his book will be the first step towards a complete review of the relevant material, carried out by a team of experts: the development of the subject, however, points to the need for a series of yearbooks rather than for a comprehensive treatise. 